\title{
Impact of palbociclib combinations on treatment of advanced estrogen receptor-positive/human epidermal growth factor 2-negative breast cancer
}

This article was published in the following Dove Press journal:

OncoTargets and Therapy

II October 2016

Number of times this article has been viewed

\section{Katalin Boér}

Department of Medical Oncology, Szent Margit Hospital, Budapest, Hungary

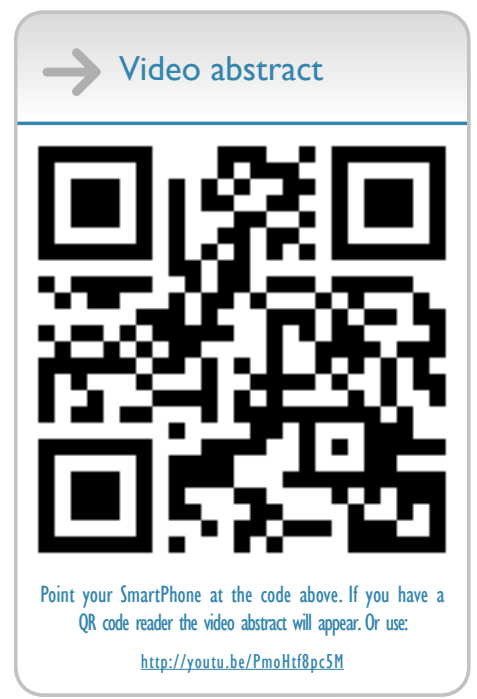

Correspondence: Katalin Boér Department of Medical Oncology, Szent Margit Hospital, No I 32 Bécsi Street, Budapest 1032, Hungary

Tel +36 I 2502420

Fax +36 | 3686665

Email katalin.boer@t-online.hu

\begin{abstract}
Breast cancer is a heterogeneous disease with multiple subgroups based on clinical and molecular characteristics. For the largest subgroup of breast cancers, hormone receptorpositive/human epidermal growth factor 2 (HER2)-negative tumors, hormone treatment is the mainstay of therapy and is likely to result in significant improvement in disease outcomes. However, some of these cancers demonstrate de novo or acquired resistance to endocrine therapy. Despite intensive research to develop new strategies to enhance the efficacy of currently available treatment options for hormone receptor-positive breast cancer, progress has been slow, and there were few advances for a period of 10 years. In 2012, a new molecularly targeted therapeutic strategy, inhibition of mammalian target of rapamycin with everolimus, was introduced into clinical practice. Everolimus, in combination with a steroidal aromatase inhibitor, exemestane, resulted in an increase in progression-free survival, but not overall survival in patients with estrogen receptor (ER)+ve advanced disease who had progressed on hormone therapy. In 2015, the first cyclin-dependent kinases 4/6 (CDK4/6) inhibitor, palbociclib, received accelerated US Food and Drug Administration approval for use in combination with letrozole for the treatment of postmenopausal ER+ve/HER2-ve advanced breast cancer as initial, endocrine-based therapy. The addition of palbociclib to endocrine therapy resulted in longer progression-free survival than letrozole alone. One year later, palbociclib received a new indication, use in combination with fulvestrant, in both premenopausal and postmenopausal females with advanced breast cancer of the same subtype with disease progression following endocrine therapy. Adding palbociclib to fulvestrant resulted in a significantly increased median progression-free survival compared to fulvestrant monotherapy. These new combination regimens of palbociclib with endocrine agents represent an important addition to the therapeutic armamentarium in ER+ve/HER2-ve advanced and metastatic breast cancer.
\end{abstract}

Keywords: breast cancer, endocrine treatment, CDK4/6 inhibitor, palbociclib

\section{Introduction}

Breast cancer is a heterogeneous disease, with multiple molecular profiles. Tumors can be classified according to the expression of the estrogen receptor (ER), progesterone receptor, and human epidermal growth factor 2 (HER2, also known as HER2/neu) receptor status into HER2+ve, ER+ve/HER2-ve, and triple-negative clinical subtypes. ${ }^{1}$ ER is expressed in $\sim 70 \%$ of all breast cancers and endocrine therapies are the mainstay of treatment for these tumors. ${ }^{2}$

According to guidelines, hormone therapy is a major therapeutic option in locally advanced and metastatic ER+ve/HER2-ve breast cancer. Sequential hormone therapy (alone or in combination) should be used as initial treatment, except in cases 
of immediately life-threatening disease, or with extensive visceral or symptomatic metastases. Until recently, the only hormonal strategies in clinical use were tamoxifen, aromatase inhibitors, and fulvestrant used alone. ${ }^{3,4}$ Even though endocrine therapy is the most effective treatment for ER+ve/HER2-ve tumors, up to a quarter may show de novo resistance to hormone therapies or acquired resistance during the course of disease. For this reason, efforts have been made to identify new therapeutic strategies to overcome endocrine resistance and to enhance the efficacy of endocrine treatments by combining hormone therapy with other targeted treatment approaches, such as small-molecule inhibitors. In this context, targeting the mammalian target of rapamycin with everolimus in combination with exemestane resulted in improving progression-free survival (PFS) in patients with hormone receptor (HR)+ve breast cancer who had progressed on an aromatase inhibitor. ${ }^{5,6}$ In addition, data from several clinical trials suggest that inhibitors of the cyclin-dependent kinases 4 and $6(\mathrm{CDK} 4 / 6)$ are also able to delay or reverse resistance to endocrine therapy. The first-in-class CDK4/6 inhibitor, palbociclib, has been introduced into clinical practice in 2015 for the treatment of ER+ve/HER2-ve locally advanced or metastatic breast cancer. ${ }^{7-10}$

\section{Inhibition of CDK4/6}

The cell cycle is driven by an interplay between cyclins and their associated cyclin-dependent kinases, a family of serine/threonine kinases that interact with cyclins, and have an important role in cell division. Despite the large number of CDKs and cyclins, only a few have been strongly implicated in breast cancer pathogenesis. Oncogenic signals in HR+ve breast cancer promote expression of cyclin D1 and activate CDK4/6, which could result in breast cancer proliferation. Newly synthesized cyclin D1 forms activating complexes with CDK4/6, which initiate phosphorylation of retinoblastoma gene product (pRb). ${ }^{11-13}$ The cyclin D-CDK4/6-Rb pathway, which is downstream of many mitogenic cascades, controls the cell cycle restriction point and is commonly dysregulated in breast carcinoma. This pathway became an important target for drug development and its alteration can occur by different mechanisms, such as CDK mutations, inactivation of p16, and overexpression of the cyclin D1 gene. ${ }^{14}$ In ER+ve breast cancer, both steroid and peptide growth factors drive proliferation through cyclin D-CDK4/6 activation, which results in the hyperphosphorylation of $\mathrm{pRb}$. In the presence of hyperphosphorylated pRb, the transcription factor E2 (E2F) is released and the cell progresses through the $\mathrm{S}$ phase of

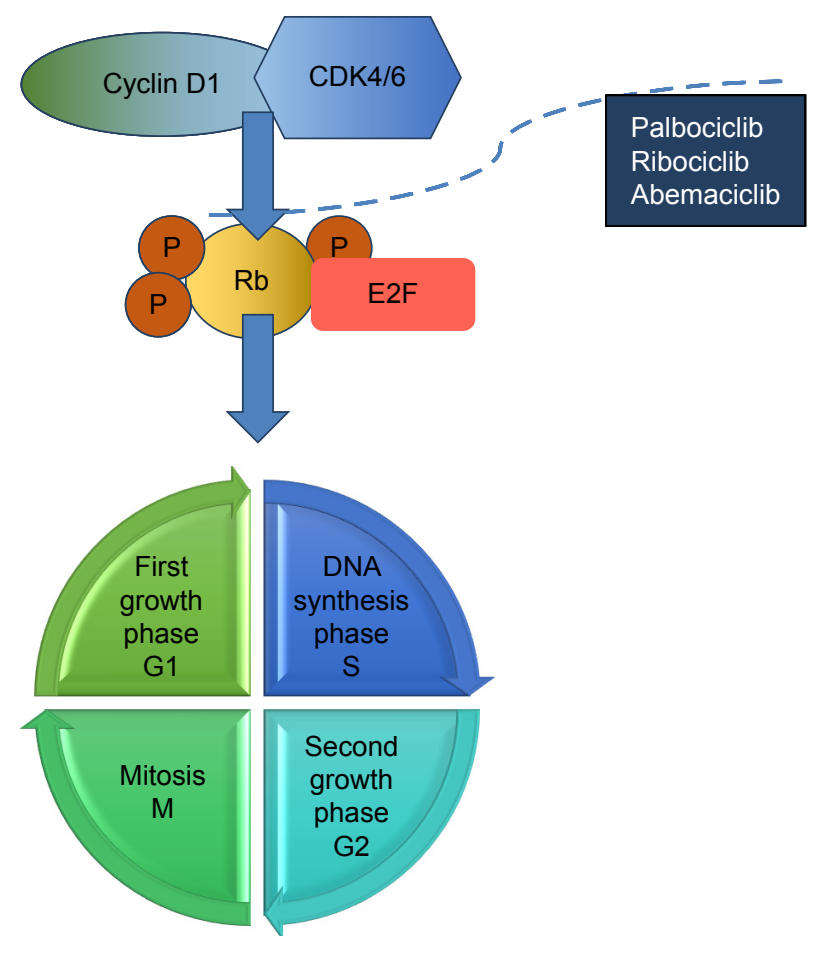

Figure I CDK4/CDK6 inhibitors mechanism of action.

Notes: In ER+ve/HER2-ve breast cancer oncogenic signals promote expression of cyclin DI and activate CDK4/CDK6, which could result in breast-cancer proliferation. Cyclin DI forms activating complexes with CDK4/6, which initiate $\mathrm{pRb}$. In the presence of hyperphosphoryated pRb, the E2F is released and the cell cycle goes through $S$ phase. Small molecule kinase inhibitors of CDK4/6 (palbociclib, ribociclib, abemaciclib) block the hyperphosphorylation of $\mathrm{pRb}$ and induce $\mathrm{GI}$ cell arrest. ${ }^{7}$

Abbreviations: CDK, cyclin-dependent kinase; E2F, transcription factor E2; ER, estrogen receptor; HER2, human epidermal growth factor 2; Rb, retinoblastoma; $\mathrm{pRb}$, Retinoblastoma protein; P, phosphate.

the cycle (Figure 1). Small-molecule inhibitors of CDK4/6 block the hyperphosphorylation of $\mathrm{pRb}$ and cause $\mathrm{G} 1$ cell cycle arrest. Greater knowledge of the interplay between the steroid hormone pathway, peptide growth factor signaling, and CDK biology is likely to broaden our understanding of the mechanisms of endocrine resistance. ${ }^{7,15}$

\section{Therapeutic CDK4/6 inhibition in breast cancer}

In early studies, the first-generation of less selective CDK inhibitors, such as flavopiridol and seliciclib showed minimal activity and had several dose-limiting toxicities, including unacceptably high rates of neutropenia, hyperglycemia, cardiac, and pulmonary dysfunction. Recently, small-molecule inhibitors specific for CDK $4 / 6$ have entered clinical testing. ${ }^{7,8}$ Currently, three oral selective CDK4/6 inhibitors are at different stages of clinical development: palbociclib (PD-0332991), ribociclib (LEE011), and 
Table I Phase II/III clinical studies with CDK4/6 inhibitors in breast cancer

\begin{tabular}{|c|c|c|c|c|c|}
\hline Clinical trial & Trial setting & Primary endpoint & Combination & $\mathbf{N}$ & Phase \\
\hline \multicolumn{6}{|l|}{ Abemaciclib } \\
\hline neoMONARCH ${ }^{29}$ & Neoadjuvant & Biomarker & Anastrozole & 220 & II \\
\hline MONARCH-I ${ }^{30}$ & Metastatic & Response & Single agent & 128 & II \\
\hline MONARCH-2 $2^{31}$ & Metastatic & PFS & Fulvestrant & 630 & III \\
\hline MONARCH-3 $3^{32}$ & Metastatic & PFS & Nonsteroidal Al & 450 & III \\
\hline NCT0230802033 & Brain metastasis & Response & Single agent & 120 & II \\
\hline \multicolumn{6}{|l|}{ Ribociclib } \\
\hline MONALEESA- ${ }^{34}$ & Neoadjuvant & Pharmacodynamic & Letrozole & 120 & II \\
\hline MONALEESA-2 $2^{35}$ & Metastatic & PFS & Letrozole & 650 & III \\
\hline MONALEESA-336 & Metastatic & PFS & Fulvestrant & 660 & III \\
\hline \multirow{2}{*}{ MONALEESA $-7^{37}$} & Metastatic & PFS & Tamoxifen & 660 & III \\
\hline & (premenopausal) & & Non-steroidal Al & & \\
\hline \multicolumn{6}{|l|}{ Palbociclib } \\
\hline PALOMA-I/TRIO-I $18^{19}$ & Metastatic & PFS & Letrozole & 165 & II \\
\hline PALOMA-2 $2^{38}$ & Metastatic & PFS & Letrozole & 450 & III \\
\hline PALOMA-321 & Metastatic & PFS & Fulvestrant & 417 & III \\
\hline PALOMA-4 ${ }^{27}$ & Metastatic & PFS & Letrozole & 330 & III \\
\hline PEARL ${ }^{39}$ & Metastatic & PFS & Exemestane vs capecitabine & 348 & III \\
\hline PENELOPE-B ${ }^{23}$ & High risk adjuvant & iDFS & Antihormonal & 800 & III \\
\hline PALLET ${ }^{25}$ & Neoadjuvant & Biomarker cCR & Letrozole & 306 & II \\
\hline PARSIFAL ${ }^{28}$ & Metastatic & PFS & Letrozol or Fulvestrant & 304 & III \\
\hline FLIPPER ${ }^{40}$ & Metastatic & PFS & Letrozol or Fulvestrant & 190 & II \\
\hline PALLAS $^{24}$ & Adjuvant & iDFS & Antihormonal & 4,600 & III \\
\hline NeoPAL ${ }^{26}$ & Neoadjuvant & $\mathrm{RCB}$ & Letrozole vs FEC-3 & 306 & II \\
\hline
\end{tabular}

Abbreviations: Al, aromatase inhibitor; CDK, cyclin D-cyclin-dependent kinase; cCR, clinical complete response, FEC, fluorouracil, epirubicin, cyclophosphamide; iDFS, invasive disease-free interval, N, number of patients; PFS, progression-free survival, RCB, residual cancer burden.

abemaciclib (LY2835219). Of these, palbociclib is the most advanced in development (Table 1).

\section{Palbociclib: preclinical data}

Palbociclib (PD-0332991) is an orally bioavailable CDK4/6 inhibitor with IC50 of $0.01 \mu \mathrm{M}$ in vitro and high selectivity for CDK4/6 compared with other CDKs. In preclinical studies, palbociclib has been shown to target CDK4/6 specifically, with potent inhibition of tumor cell proliferation by $\mathrm{G} 1$ arrest, leading to dephosphorylation of $\mathrm{pRb}$, a decrease of E2F-dependent gene expression and potent inhibition of tumor cell proliferation through arrest in G1. ${ }^{16,17}$ Moreover, palbociclib is completely inactive in $\mathrm{pRb}$-negative tumor cell lines and xenografts. The pioneering work of Finn et al documented the activity of palbociclib in reducing proliferation of $\mathrm{ER}+\mathrm{ve}$ breast cancer cell lines by blocking the progression of cells from $\mathrm{G} 1$ into the $\mathrm{S}$ phase of the cell cycle. They also showed that ER+ve or HER2-amplified cell lines subtypes were most sensitive to palbociclib inhibition while cell lines from other nonluminal subtypes were most resistant. In addition, synergy was demonstrated between palbociclib and tamoxifen in growth inhibition and the drug showed activity in a model of acquired tamoxifen resistence. ${ }^{7,18}$

\section{Clinical efficacy of palbociclib in combination with hormone therapy Palbociclib in combination with letrozole as initial endocrine-based therapy}

Palbociclib in combination with letrozole received accelerated US Food and Drug Administration approval in February 2015, as initial therapy for postmenopausal females with HR+ve/HER2-ve advanced breast cancer, based on the very favorable PFS results of PALOMA 1 clinical trial. PALOMA-1 (NCT00721409) was a randomized, multicentre, Phase I/II study, in which postmenopausal patients with HR+ve/HER2-ve advanced breast cancer received either the combination of palbociclib and letrozole, or letrozole alone. A total of 165 postmenopausal females with stage IV breast cancer or inoperable locally recurrent disease who had not received any systemic treatment for advanced disease were enrolled in the trial. In the combination arm, treatment consisted of palbociclib $125 \mathrm{mg} /$ day for $21 \mathrm{con}-$ secutive days followed by 7 days off treatment and letrozole $2.5 \mathrm{mg} /$ day continuously. Initially, the study consisted of two parts: in part I enrollment was based on HR and HER2 status alone and the efficacy, safety, and tolerability of the 
combination was assessed. In part II of the trial, eligibility criteria included the presence of cyclin D1 amplification and/ or p16 loss, in addition to HR and HER2 status. At the final PFS analyses, median PFS was 10.2 months for letrozole alone and 20.2 months with the palbociclib and letrozole combination (hazard ratio [HR] $0.488,95 \%$ confidence interval [CI] 0.319-0.748, 1-sided $P=0.0004)$. In both cohorts, significant clinical benefit was observed from the addition of palbociclib to letrozole. However, cyclin D1 status and p16 expression did not add further predictive value for the efficacy of palbociclib over ER status alone. There was a trend toward increased overall survival (OS) for the combination arm in comparison to the monotherapy group (37.5 months vs 33.3 months, HR 0.813 ; 95\% CI 0.492-1.345, 2-sided $P=0.4210$ ), but significance was not reached, probably due to the small number of patients. The addition of palbociclib to endocrine therapy resulted in improvement in the objective response rate $(43 \%$ vs $33 \%, 1$-sided $P=0.1347)$ and the clinical benefit rate ( $81 \%$ vs $58 \%, 1$-sided $P=0.0009$ ), which were secondary endpoints. Efficacy subgroup analyses showed consistent clinical benefit of palbociclib in combination with letrozole, regardless of age, tumor characteristics, prior systemic therapy in the neoadjuvant/adjuvant setting and presence or absence of visceral disease..$^{19,20}$

\section{Palbociclib in combination with fulvestrant following endocrine therapy}

Food and Drug Administration approval of palbociclib was extended in February 2016, when the drug received a new indication for use in combination with fulvestrant, based on the results of the PALOMA-3 (NCT01942135) clinical trial. In this Phase III study, patients were randomized to receive palbociclib (125 mg/day orally for 21 days, followed by 7 days off) and fulvestrant (500 mg intramuscularly every 14 days for the first three injections and then every 28 days) or placebo and fulvestrant. In contrast to PALOMA-1, premenopausal or perimenopausal patients were also eligible; they received goserelin for the duration of study treatment. PALOMA-3 was terminated early, when the primary endpoint had been met at the preplanned interim analysis. The final PFS was 9.5 months in the group treated with the combination of palbociclib and fulvestrant, and 4.6 months with placebo-fulvestrant (hazard ratio $0.46,95 \%$ CI $0.36-0.59$, $P<0.0001) .{ }^{21}$ The PFS results were consistent in subgroup analyses, across various demographic and prognostic factors. Moreover, the relative difference in PFS between palbociclib and placebo was similar in premenopausal, perimenopausal, and postmenopausal patients. The overall objective response rates were $10.4 \%$ with palbociclib-fulvestrant and $6.3 \%$ with placebo-fulvestrant $(P=0.16)$. The rate of clinical benefit, defined as response or prolonged stable disease, was higher with the combination of palbociclib and endocrine therapy than with placebo-fulvestrant $(34.0 \%$ vs $19.0 \%$, $P<0.001) .{ }^{22}$ At the time of the first interim analysis, the OS data were immature.

\section{Palbociclib safety profile: adverse events of special interest}

In two, randomized, international studies (PALOMA-1 and PALOMA-3) palbociclib in combination with endocrine therapy (letrozole or fulvestrant) was an effective and well-tolerated treatment for patients with advanced ER+ve/ HER2-ve breast cancer, with manageable toxicity. In both studies, adverse events of special interest were neutropenia ( $75 \%$ vs $5 \%$ ), leukopenia ( $43 \%$ vs $3 \%$ ), fatigue $(41 \%$ vs $23 \%$ ), and anemia ( $35 \%$ vs $7 \%$ ). Neutropenia was reported in $75 \%$ of patients in PALOMA-1, whereas this side-effect occurred in $83 \%$ of cases in PALOMA-3. Despite the high rate of leukopenia and neutropenia, febrile neutropenia was reported in only $\sim 1 \%$ of patients exposed to palbociclib, and did not lead to serious clinical consequences. ${ }^{19,21}$ Neutropenia is believed to be due to the cytostatic effect of palbociclib on bone marrow progenitor cells. ${ }^{7}$ According to the product label, complete blood counts are required prior to starting palbociclib therapy, at the beginning of each cycle, on day 14 of the first two cycles, then as clinically indicated. For patients developing grade 3 or 4 neutropenia, dose interruption, dose reduction, or delay in starting subsequent treatment cycles is recommended. ${ }^{10}$

\section{Future directions: palbociclib in early-stage breast cancer}

Based on the therapeutic advantage observed with the addition of palbociclib to hormone therapy in metastatic breast cancer, evaluation in early-stage breast disease is a logical next step. Currently, two large Phase III studies, PENELOPE-B and PALLAS, are ongoing, evaluating the feasibility of adding palbociclib to hormone therapy as adjuvant treatment in early breast cancer. ${ }^{23,24}$ Two other clinical trials (PALLET and NeoPal) have been designed to evaluate the role of CDK4/6 inhibition with palbociclib as neoadjuvant treatment. ${ }^{25,26}$

\section{Expert opinion}

Despite advances in the treatment of ER+ve/HER2-ve breast cancer, there is a high incidence of recurrence and progression on available standard therapies. Personalizing the use of anticancer treatments is a major focus in clinical 
practice. Deregulation of the cyclin D-CDK4/6-Rb pathway is commonly seen in ER+ve breast cancer and this observation led to the identification of CDK $4 / 6$ as an attractive new target for treatment. The development of CDK $4 / 6$ inhibitors and the introduction of palbociclib, the first in this class, into clinical practice, represent an important addition to the therapeutic armamentarium in metastatic breast cancer.

Adding palbociclib to endocrine therapy significantly extended PFS in comparison with endocrine therapy in the first and subsequent lines of treatment for ER+ve/HER2-ve advanced and metastatic breast cancer. Moreover, palbociclib has shown a consistent clinical efficacy and safety profile in combination with letrozole or fulvestrant in this subtype of breast cancer. In first-line treatment in postmenopausal advanced breast cancer, the addition of palbociclib to letrozole was associated with significantly longer PFS than letrozole alone (20.2 vs 10.2 months). In addition, the combination of palbociclib and fulvestrant resulted in a significantly longer PFS (9.5 vs 4.6 months) compared with fulvestrant alone when used in later lines of treatment in both pre- and postmenopausal patients who had progressed on previous endocrine therapy. Recently, the primary results of a confirmatory Phase III trial, PALOMA-2 were presented; these expand and confirm the significant clinical benefit and safety of the palbociclib-letrozole combination in ER+ve/HER2-ve advanced breast cancer. OS data are not yet available or mature, but the clinically relevant activity of palbociclib resulting in more than doubling of the PFS is sufficient for it to be used in patients carefully selected based on clinical characteristics to identify those who are likely to benefit most. In addition, the observed PFS prolongation raises the possibility of improvement in OS, which could ultimately lead to ER+ve/HER2-ve advanced breast cancer becoming a chronic disease. The results of PALOMA-1 support the preclinical observation that ER positivity is the best biomarker so far to select patients likely to benefit from CDK4/6 inhibition. Overall, the combination of palbociclib and endocrine therapy is a well-tolerated treatment, with leukopenia and neutropenia being the most common side-effects.

There are several therapeutic choices available for ER+ve/HER2-ve advanced breast cancer, but adding palbociclib to hormone therapy is an option with strong efficacy data. Moreover, at present, based on the remarkable PFS prolongation the combination of palbociclib and hormone therapy can be used in well-selected patients to delay the need to initiate cytotoxic chemotherapy.

Two other CDK4/6 inhibitors, ribociclib and abemaciclib, are in clinical development with ongoing clinical trials in similar combinations and indications, as well as other indications. CDK4/6 inhibitors may have a role in the adjuvant setting due to their good clinical activity and the low, tolerable, and predictable side effects. Studies are also ongoing to evaluate CDK4/6 inhibitors as neoadjuvant and adjuvant treatment. There are several open questions about the role of CDK4/6 inhibitors in daily practice, such as the increased treatment costs, the optimal place in the treatment continuum, and identification of predictive markers for routine patient selection.

\section{Disclosure}

The author has received consulting fees from GlaxoSmithKline, Novartis, Pfizer, and Roche.

\section{References}

1. Perou CM, Sorlie T, Eisen MB, et al. Molecular portraits of human breast tumours. Nature. 2000;406(6797):747-752.

2. Perez EA. Treatment strategies for advanced hormone receptor-positive and human epidermal growth factor 2-negative breast cancer: the role of treatment order. Drug Resist Updat. 2016;24:13-22.

3. Rugo HS, Rumble RB, Burstein HJ. Endocrine therapy for hormone receptor positive metastatic breast cancer: American Society of Clinical Oncology guideline summary. J Oncol Pract. 2016;12(6):583-587.

4. Cardoso F, Costa A, Norton L, et al. ESO-ESMO 2nd international consensus guidelines for advanced breast cancer (ABC2). Breast. 2014; 23(5):489-502.

5. Osborne CK, Schiff R. Mechanisms of endocrine resistance in breast cancer. Annu Rev Med. 2011;62:233-247.

6. O'Sullivan CC. Overcoming endocrine resistance in hormone-receptor positive advanced breast cancer-the emerging role of CDK4/6 inhibitors. Int J Cancer Clin Res. 2015;2(4):2-14.

7. Finn RS, Aleshin A, Slamon DJ. Targeting the cyclin-dependent kinases (CDK) 4/6 in estrogen receptor-positive breast cancers. Breast Cancer Res. 2016;18(1):17.

8. Steger GG, Gnant M, Bartsch R. Palbociclib for the treatment of postmenopausal breast cancer - an update. Expert Opin. 2016;17(2):255-263.

9. Beaver JA, Amiri-Kordestani L, Beaver JA. FDA approval: palbociclib for the treatment of postmenopausal patients with estrogen receptor-positive, HER2-negative metastatic breast cancer. Clin Cancer Res. 2015; 21(21):4760-4766.

10. FDA approves Ibrance for postmenopausal women with advanced breast cancer. Available from: http:/www.fda.gov/NewsEvents/Newsroom/ PressAnnouncements/ucm432871.htm. Accessed January 26, 2015.

11. Hanahan D, Weinberg RA. Hallmarks of cancer: the next generation. Cell. 2011;144(5):646-674.

12. Satyanarayana A, Kaldis P. Mammalian cell-cycle regulation: several Cdks, numerous cyclins and diverse compensatory mechanisms. Oncogene. 2009;28(33):2925-2939.

13. Bertoli C, Skotheim JM, de Bruin RA. Control of cell cycle transcription during G1 and S phases. Nat Rev Mol Cell Biol. 2013;14(8):518-528.

14. Landis MW, Pawlyk BS, Li T, Sicinski P, Hinds PW. Cyclin D1-dependent kinase activity in murine development and mammary tumorigenesis. Cancer Cell. 2006;9(1):13-22.

15. Dean JL, Thangavel C, McClendon AK, Reed CA, Knudsen ES. Therapeutic CDK4/6 inhibition in breast cancer: key mechanisms of response and failure. Oncogene. 2010;29(28):4018-4032.

16. Toogood PL, Harvey PJ, Repine JT, et al. Discovery of a potent and selective inhibitor of cyclin-dependent kinase 4/6. J Med Chem. 2005; 48(7):2388-2406.

17. Fry DW, Harvey PJ, Keller PR, et al. Specific inhibition of cyclin-dependent kinase 4/6 by PD 0332991 and associated antitumor activity in human tumor xenografts. Mol Cancer Ther. 2004;3(11):1427-1438. 
18. Finn RS, Crown JP, Lang I, et al. Results of a randomized phase 2 study of PD 0332991, a cyclin-dependent kinase (CDK) 4/6 inhibitor, in combination with letrozole vs letrozole alone for first line treatment of ER+/HER2- advanced breast cancer (BC). Cancer Res. 2012; 72(24 Suppl):S1-S6.

19. Finn RS, Crown JP, Lang I, et al. The cyclin-dependent kinase $4 / 6$ inhibitor palbociclib in combination with letrozole versus letrozole alone as first-line treatment of oestrogen receptor-positive, HER2-negative, advanced breast cancer (PALOMA-1/TRIO-18): a randomised phase 2 study. Lancet Oncol. 2014;16(1):25-35.

20. Crown J, Richard SF, Ettl J, et al. Efficacy and safety of first-line palbociclib plus letrozole compared with letrozole alone in patients aged $\geq 65$ years with estrogen receptor-positive, HER2-negative advanced breast cancer: A subgroup analysis by age of the PALOMA-1/TRIO-18 trial. J Clin Oncol. 2015;33 suppl; abstr 571.

21. Turner NC, Huang Bartlett C, Cristofanilli M. Palbociclib in hormonereceptor-positive advanced breast cancer. N Engl J Med. 2015;373(17): 1672-1673.

22. Cristofanilli M, Turner NC, Bondarenko I, et al. Fulvestrant plus palbociclib versus fulvestrant plus placebo for treatment of hormonereceptor-positive, HER2-negative metastatic breast cancer that progressed on previous endocrine therapy (PALOMA-3): final analysis of the multicentre, double-blind, phase 3 randomised controlled trial. Lancet Oncol. 2016;17(4):425-439.

23. Phase III study evaluating palbociclib (PD-0332991), a cyclin-dependent kinase (CDK) 4/6 inhibitor in patients with hormone-receptorpositive, HER2-normal primary breast cancer with high relapse risk after neoadjuvant chemotherapy "PENELOPEB". Available from: https://clinicaltrials.gov/ct2/show/NCT01864746. NLM identifier: NCT01864746. Accessed August 15, 2016.

24. Palbociclib collaborative adjuvant study: a randomized phase iii trial of palbociclib with standard adjuvant endocrine therapy versus standard adjuvant endocrine therapy alone for hormone receptor positive $(\mathrm{HR}+) / \mathrm{Human}$ Epidermal Growth Factor Receptor 2 (HER2)-negative early breast cancer. Available from: https://clinicaltrials.gov/ct2/show/NCT02513394. NLM identifier: NCT02513394. Accessed August 15, 2016.

25. A phase II randomized study evaluating the biological and clinical effects of the combination of palbociclib with letrozole as neoadjuvant therapy in post-menopausal women with estrogen-receptor positive primary breast cancer. Available from: https://clinicaltrials.gov/ct2/ show/NCT02296801. NLM identifier: NCT02296801. Accessed August 15, 2016.

26. Open-label, randomized, multicenter, international, parallel exploratory phase ii study, comparing 3 FEC-3 docetaxel chemotherapy to letrozole + palbociclib combination as neoadjuvant treatment of stage II-IIIA PAM 50 ROR-defined low or intermediate risk luminal breast cancer, in postmenopausal women. Available from: https://clinicaltrials.gov/ct2/ show/NCT02400567. NLM identifier: NCT02400567. Accessed August $15,2016$.

27. A multicenter, randomized, double-blind phase 3 study of palbociclib (oral CDK 4/6 inhibitor) plus letrozole versus placebo plus letrozole for the treatment of previously untreated asian postmenopausal women with ER (+), HER2 (-) advanced breast cancer. Available from: https://clinicaltrials.gov/ct2/show/NCT02297438. NLM identifier: NCT02297438. Accessed August 15, 2016.

28. A randomized, multicenter, open-label, phase II trial to evaluate the efficacy and safety of plabociclib in combination with fulvestrant or letrozole in patients with HER2 negative, ER+ metastatic breast cancer. Available from: https://clinicaltrials.gov/ct2/show/NCT02491983. NLM identifier: NCT02491983. Accessed August 15, 2016.

29. neoMONARCH: a phase 2 neoadjuvant trial comparing the biological effects of 2 weeks of abemaciclib (LY2835219) in combination with anastrozole to those of abemaciclib monotherapy and anastrozole monotherapy and evaluating the clinical activity and safety of a subsequent 14 weeks of therapy with abemaciclib in combination with anastrozole in postmenopausal women with hormone receptor positive, HER2 negative breast cancer. Available from: https://clinicaltrials.gov/ct2/show/NCT02441946. NLM identifier: NCT02441946. Accessed August 15, 2016.
30. A phase 2 study Of LY2835219 for patients with previously treated hormone receptor positive, HER2 negative metastatic breast cancer. Available from: https://clinicaltrials.gov/ct2/show/NCT02102490. NLM identifier: NCT02102490. Accessed August 15, 2016.

31. MONARCH 2: a randomized, double-blind, placebo-controlled, phase 3 study of fulvestrant with or without abemaciclib, a CDK4/6 inhibitor, for women with hormone receptor positive, HER 2 negative locally advanced or metastatic breast cancer. Available from: https://clinicaltrials.gov/ ct2/show/NCT02107703. NLM identifier: NCT02107703. Accessed August 15, 2016.

32. Goetz MP, Toi M, Klise S, et al. MONARCH 3: A randomized phase III study of anastrozole or letrozole plus abemaciclib, a CDK4/6 inhibitor, or placebo in first-line treatment of women with HR+, HER2-locoregionally recurrent or metastatic breast cancer (MBC). J Clin Oncol. 2015;33:suppl; abstr TPS624. Accessed August 15, 2016.

33. A phase 2 study of abemaciclib in patients with brain metastases secondary to hormone receptor positive breast cancer, non-small cell lung cancer, or Melanoma. Available from: https://clinicaltrials.gov/ ct2/show/NCT02308020. NLM identifier: NCT02308020. Accessed August 15, 2016.

34. A Randomized pre-surgical pharmacodynamics study to assess the biological activity of LEE011 plus letrozole versus single agent letrozole in primary breast cancer. Available from: https://clinicaltrials.gov/ct2/ show/NCT01919229. NLM identifier: NCT01919229. Accessed August 15, 2016.

35. A randomized double-blind, placebo-controlled study of LEE011 in combination with letrozole for the treatment of postmenopausal women with hormone receptor positive, HER 2 negative, advanced breast cancer who received no prior therapy for advanced disease. Available from: https:/clinicaltrials.gov/ct2/show/NCT01958021. NLM identifier: NCT01958021. Accessed August 15, 2016.

36. A randomized double-blind, placebo-controlled study of ribociclib in combination with fulvestrant for the treatment of men and postmenopausal women with hormone receptor positive, HER2-negative, advanced breast cancer who have received no or only one line of prior endocrine treatment. Available from: https://clinicaltrials.gov/ct2/show/NCT02422615. NLM identifier: NCT02422615. Accessed August 15, 2016.

37. A phase III randomized, double-blind, placebo-controlled study of LEE011 or placebo in combination with tamoxifen and goserelin or a Non-steroidal Aromatase Inhibitor (NSAI) and goserelin for the treatment of premenopausal women with hormone receptor positive, HER2-negative, advanced breast cancer. Available from: https:/clinicaltrials.gov/ct2/show/NCT02278120. NLM identifier: NCT02278120. Accessed August 15, 2016.

38. Finn RS, Martin M, Rugo HS, et al. PALOMA-2: Primary results from a phase III trial of palbociclib (P) with letrozole (L) compared with letrozole alone in postmenopausal women with ER+/HER2-advanced breast cancer (ABC). J Clin Oncol. 2016;34:suppl; abstr 507.

39. Phase III study of palbociclib (PD-0332991) in combination with exemestane versus chemotherapy (capecitabine) in hormonal receptor (HR) positive/HER2 negative metastatic breast cancer (MBC) patients with resistance to non-steroidal aromatase inhibitors (PEARL). Available from: http://clinicaltrials.gov/ct2/show/NCT02028507. NLM identifier: NCT02028507. Accessed August 15, 2016.

40. A randomized, double-blind, parallel-group, multicentre, phase ii study to compare the efficacy and tolerability of fulvestrant (faslodextm) $500 \mathrm{mg}$ with placebo and fulvestrant (faslodextm) $500 \mathrm{mg}$ in combination with PD-0332991 (Palbociclib) as first line treatment for postmenopausal women with hormone receptor-positive metastatic breast cancer, who have completed at least 5 years of adjuvant endocrine therapy and remained disease free for more than 12 months following its completion or have "de novo" metastatic disease. "The FLIPPER Study". Available from: https://clinicaltrials.gov/ct2/show/NCT02690480. NLM identifier: NCT02690480. Accessed August 15, 2016. 
OncoTargets and Therapy

\section{Publish your work in this journal}

OncoTargets and Therapy is an international, peer-reviewed, open access journal focusing on the pathological basis of all cancers, potential targets for therapy and treatment protocols employed to improve the management of cancer patients. The journal also focuses on the impact of management programs and new therapeutic agents and protocols on The manuscript management system is completely online and includes a very quick and fair peer-review system, which is all easy to use. Visit http://www.dovepress.com/testimonials.php to read real quotes from published authors.

Submit your manuscript here: http://www.dovepress.com/oncotargets-and-therapy-journal 